\title{
Probing dark photons with plasma haloscopes
}

\author{
Graciela B. Gelmini®, ${ }^{1}$ Alexander J. Millar, ${ }^{2,3}$ Volodymyr Takhistov, ${ }^{1}$ and Edoardo Vitagliano ${ }^{1, *}$ \\ ${ }^{1}$ Department of Physics and Astronomy, University of California, \\ Los Angeles Los Angeles, California, 90095-1547, USA \\ ${ }^{2}$ The Oskar Klein Centre for Cosmoparticle Physics, Department of Physics, Stockholm University, \\ AlbaNova, 10691 Stockholm, Sweden \\ ${ }^{3}$ Nordita, KTH Royal Institute of Technology and Stockholm University, \\ Roslagstullsbacken 23, 10691 Stockholm, Sweden
}

(Received 19 June 2020; accepted 22 July 2020; published 7 August 2020)

\begin{abstract}
Dark photons (DPs) produced in the early Universe are well-motivated dark matter (DM) candidates. We show that the recently proposed tunable plasma haloscopes are particularly advantageous for DP searches. While in-medium effects suppress the DP signal in conventional searches, plasma haloscopes make use of metamaterials that enable resonant absorption of the DP by matching its mass to a tunable plasma frequency and thus enable efficient plasmon production. Using thermal field theory, we confirm the in-medium DP absorption rate within the detector. This scheme allows us to competitively explore a significant part of the DP DM parameter space in the DP mass range of 6-400 $\mu \mathrm{eV}$. If a signal is observed, the observation of a daily or annual modulation of the signal would be crucial to clearly identify the signal as due to DP DM and could shed light on the production mechanism.
\end{abstract}

DOI: 10.1103/PhysRevD.102.043003

\section{INTRODUCTION}

Uncovering the nature of dark matter (DM), the predominant constituent of matter in the Universe, remains an elusive target and subject of significant scientific efforts (see, e.g., Ref. [1] for a review). While a lot of attention has been devoted to exploring DM composed of weakly interacting massive particles (WIMPs), another generic possibility is DM composed of very light $(\ll \mathcal{O}(\mathrm{eV}))$ bosonic particles that exhibit classical fieldlike behavior.

Dark (hidden) photons (DPs) constitute a prototypical example of spin-1 bosonic light DM (e.g., [2-4]). Associated with an additional U(1) dark sector gauge symmetry, they appear in minimal extensions of the Standard Model (SM) and are also well motivated by fundamental considerations (e.g., [5]). Analogously to axions [6-8], DPs can be readily produced in the early Universe through the misalignment mechanism [9-13] (other production channels, such as inflationary fluctuations [14,15] or decays of precursor fields [16-20], are also possible). Depending on its production history, the resulting DP vector field may or may not

\footnotetext{
*Corresponding author. edoardo@physics.ucla.edu

Published by the American Physical Society under the terms of the Creative Commons Attribution 4.0 International license. Further distribution of this work must maintain attribution to the author(s) and the published article's title, journal citation, and DOI. Funded by SCOAP .
}

have a preferred direction of alignment at present time within our Galaxy.

A central pillar in experimental efforts to search for the DM are direct detection experiments that attempt to measure the energy deposited within a detector by interactions of DM in the Milky Way halo passing through it. Light DM with mass significantly smaller than $\mathcal{O}(\mathrm{GeV})$ will not deposit sufficient nuclear recoil energy within conventional ton-scale direct detection experiments (e.g., [21-23]) with keV-level thresholds optimized toward WIMP searches, focusing on electroweak mass scales. Hence, other types of searches are required to explore it.

For light DM, DPs or axions/axionlike particles (we refer to these generically as "axions"), sensitivity to $\mathcal{O}(\mathrm{eV})$ mass scales can be gained within large direct detection experiments searching for ionization signals due to DM absorption by bound electrons [24,25]. Detection proposals based on small $\mathcal{O}(\mathrm{meV})$ band-gap materials envision good sensitivity for light DM with masses above the gap, allowing for efficient quasiparticle excitation (e.g., phonons), including superconductors [26,27], graphene [28], Dirac materials [29,30], superfluid helium [31-35], and polar materials [36]. Axion haloscope experiments (see, e.g., Ref. [37] for a review) can typically also efficiently probe DPs, including setups based on cavity resonators (e.g., [38-44]) and dielectrics $[45,46]$ as well as dish antennas [47-51].

In-medium effects within detectors themselves suppress DP interactions and thus present a major limiting factor for experimental searches. If the DM interaction is on resonance with quasiparticle production within the material, 
such as plasmons, these effects can be mitigated. In a recent study [52], a novel tunable plasma haloscope for axion detection has been proposed. While the more conventional axion haloscope cavity searches for axions coupled to photons rely on resonant axion-photon conversion associated with cavity frequency modes, the plasma haloscope takes advantage of novel metamaterial properties to consider axion-plasmon resonant conversion. The tunable plasma frequency that resonantly matches the axion mass is not directly associated with the dimensions of the detector in plasma haloscopes and allows to efficiently cover a large axion parameter space.

In this work we show that plasma haloscopes constitute particularly advantageous testing grounds for DPs. Plasmons have been extensively discussed in relation to dark sectors within astrophysical environments (e.g., [4,53-55]), the early Universe (e.g.. [56]) and more recently in the context of "inelastic" nuclear recoils from heavy $\sim \mathrm{MeV}-\mathrm{GeV} \mathrm{DM}$ within the more conventional direct detection experiments [57,58].

While DP-plasmon resonances have been considered in astrophysical environments (e.g., [54,55]), they have not been discussed in laboratory settings. Utilizing a DP-plasmon resonance with a tunable frequency, plasma haloscopes allow to take full advantage of in-medium effects that often plague DP searches, and thus significantly enhance detection sensitivity.

\section{DARK PHOTON IN-MEDIUM RESONANCE}

The low-energy effective Lagrangian due to the presence of a gauge boson $X$ of a hidden sector $\mathrm{U}(1)$ symmetry that kinetically mixes (e.g., $[10,24,59,60])$ with the ordinary photon $A$ is

$$
\begin{aligned}
\mathcal{L} \supset & -\frac{1}{4} F_{\mu \nu} F^{\mu \nu}-\frac{1}{4} X_{\mu \nu} X^{\mu \nu}+\frac{\sin \alpha}{2} F^{\mu \nu} X_{\mu \nu} \\
& +e J_{\mathrm{EM}}^{\mu} A_{\mu}+\frac{m_{X}^{2} \cos ^{2} \alpha}{2} X^{\mu} X_{\mu},
\end{aligned}
$$

where $F_{\mu \nu}, X_{\mu \nu}$ denote the fields strengths of the SM photon and the DP, respectively, $J_{\mathrm{EM}}^{\mu}$ is the electromagnetic current, $m_{X}$ is the DP mass ${ }^{1}$ after neglecting terms of order $\alpha^{2}$ (see below) and $\sin \alpha$ is the kinetic mixing parameter. The kinetic mixing term can be removed by diagonalization through $\tilde{A}=A \cos \alpha, \tilde{X}=X-\sin \alpha A$. In the interaction $(\tilde{A}, \tilde{X})$ basis, the effective Lagrangian is

$$
\begin{aligned}
\mathcal{L} \supset & -\frac{1}{4} \tilde{F}_{\mu \nu} \tilde{F}^{\mu \nu}-\frac{1}{4} \tilde{X}_{\mu \nu} \tilde{X}^{\mu \nu}+\frac{e}{\cos \alpha} J_{\mathrm{EM}}^{\mu} \tilde{A}_{\mu} \\
& +\frac{m_{X}^{2} \cos ^{2} \alpha}{2}\left(\tilde{X}^{\mu} \tilde{X}_{\mu}+2 \chi \tilde{X}_{\mu} \tilde{A}^{\mu}+\chi^{2} \tilde{A}^{\mu} \tilde{A}_{\mu}\right),
\end{aligned}
$$

\footnotetext{
${ }^{1}$ The DP mass can be generated via the Higgs or the Stueckelberg mechanisms.
}

where $\chi \equiv \tan \alpha$ and $\tilde{A}, \tilde{X}$ denote the photon produced in electromagnetic interactions and the DP sterile state, respectively. In the interaction basis the electromagnetic coupling gets renormalized to $(e / \cos \alpha)$ and there are $\tilde{A}-\tilde{X}$ photon-DP oscillations due to mass-mixing.

Neglecting $\mathcal{O}\left(\chi^{2}\right)$ terms and dropping the tilde, we have

$$
\begin{aligned}
\mathcal{L} \supset & -\frac{1}{4} F_{\mu \nu} F^{\mu \nu}-\frac{1}{4} X_{\mu \nu} X^{\mu \nu}+e J_{\mathrm{EM}}^{\mu} A_{\mu} \\
& +\frac{m_{X}^{2}}{2}\left(X^{\mu} X_{\mu}+2 \chi X_{\mu} A^{\mu}\right),
\end{aligned}
$$

which gives the wave equation in vacuum (in momentum space)

$$
-K^{2} A^{\nu}=\chi m_{X}^{2} X^{\nu}
$$

where we defined the four momentum $K=(\omega, \mathbf{k})$. Here we have used the Fourier expansion for a free field with the energy $\omega=+\sqrt{|\mathbf{k}|^{2}+m_{X}^{2}}$. We treat the fields as complex, $X_{c}^{\mu}(t, \mathbf{x})$, of which the actual fields constitute the real part $X^{\mu}=\operatorname{Re}\left\{X_{c}^{\mu}\right\}$. As in Ref. [61], we include a volume $V$ in the definition of the Fourier transform, which will later simplify the definition of the DM density in Eq. (15),

$$
X_{c}^{\mu}(t, \mathbf{x})=\sqrt{V} \int \frac{d^{3} \mathbf{k}}{(2 \pi)^{3}} X^{\mu}(\mathbf{k}) e^{-i(\omega t-\mathbf{k} \mathbf{x})} .
$$

Our use of a classical field description is justified as the state occupation number is very large (e.g., [47]).

For a DP that kinetically mixes with the SM photon, effects of in-medium propagation can be significant. Inmedium interactions can be accounted for by including a linear response (e.g., [30,53,62]),

$$
J_{\text {ind }}^{\mu}=-\Pi^{\mu \nu} A_{\nu},
$$

where $\Pi^{\mu \nu}$ is a polarization tensor. Hence, the total current coupling to photon is $e J_{\mathrm{EM}}^{\mu}+J_{\text {ind }}^{\mu}$. Let us first assume an isotropic and parity invariant medium, and later discuss the extremely anisotropic wire metamaterial scenario. In the Lorentz gauge, the polarization tensor is described by two polarization functions $\Pi_{T}$ and $\Pi_{L}$, for transverse and longitudinal excitations (e.g., [24])

$$
\begin{aligned}
\Pi^{\mu \nu} & \equiv i e^{2}\left\langle J_{\mathrm{EM}}^{\mu} J_{\mathrm{EM}}^{\nu}\right\rangle=-\Pi_{T}\left(e_{+}^{\mu} e_{+}^{* \nu}+e_{-}^{\mu} e_{-}^{* \nu}\right)-\Pi_{L} e_{L}^{\mu} e_{L}^{* \nu} \\
& =\sum_{i= \pm, L} \Pi_{i} P_{i}^{\mu \nu}
\end{aligned}
$$

where $e_{L}$ and $e_{+,-}$are the longitudinal and the transverse polarization vectors, respectively, and $P_{i}^{\mu \nu}$ are projectors. Assuming $\mathbf{k}$ parallel to the $\hat{z}$ axis, the polarization vectors are 


$$
e_{L} \equiv \frac{\left(k^{2}, \omega \mathbf{k}\right)}{k \sqrt{K^{2}}} \quad \text { and } \quad e_{+,-} \equiv \frac{1}{\sqrt{2}}\left(0, \mathbf{e}_{x} \pm i \mathbf{e}_{y}\right),
$$

where $\mathbf{e}_{x}, \mathbf{e}_{y}$ are orthogonal unit vectors perpendicular to the unit vector $\mathbf{k} / k$.

The wave equation for the interaction eigenstate $A=\left(A^{0}, \mathbf{A}\right)$, in the absence of a $J_{\mathrm{EM}}^{\mu}$ reads

$$
-K^{2} A^{\nu}=-\Pi^{\mu \nu} A_{\mu}+\chi m_{X}^{2} X^{\nu} .
$$

The relationship between the dielectric tensor $\epsilon$ and the polarization tensor components is given by $[24,63]$

$$
\epsilon_{L}=1-\frac{\Pi_{L}}{\omega^{2}-k^{2}} \quad \text { and } \quad \epsilon_{T}=1-\frac{\Pi_{T}}{\omega^{2}}
$$

so that for small $k$ (small velocity of the DM) $\epsilon_{T}=\epsilon_{L}=\epsilon$. The dielectric function has real and imaginary parts, just like the polaritazion tensor. The real part is a phase shift in a travelling photon wave function, while the imaginary part is due to absorption of photons travelling in the medium. In the Drude model, typically used to describe metals, $\epsilon$ can be written as

$$
\epsilon=1-\frac{\omega_{p}^{2}}{\omega^{2}-i \omega \Gamma} \simeq 1-\frac{\omega_{p}^{2}}{\omega^{2}}+i \frac{\Gamma \omega_{p}^{2}}{\omega^{3}},
$$

where $\Gamma$ is the damping within the medium. For small $k$ we have that $\omega \simeq m_{\chi}$ and the zero-components of the fields are negligible, $A^{0} \simeq(\mathbf{k} \cdot \mathbf{A} / \omega) \simeq 0$ and $X^{0} \simeq 0$ (see Appendix of Ref. [47]). Thus, the ordinary electric field produced by DP (assuming an isotropic medium) is given by

$$
|\mathbf{E}(\mathbf{k}=\mathbf{0})|=\left|\frac{\chi m_{X}}{\epsilon} \mathbf{X}(\mathbf{k}=\mathbf{0})\right| .
$$

\section{OUTPUT POWER}

We would like to relate the $\mathbf{X}$ field in Eq. (12) with the DM energy density. First, we note that the detected electric field, as produced by DP in Eq. (12), should be the time derivative of the interaction eigenstate. ${ }^{2}$

Another remark is related to the field $\mathbf{X}$ in Eq. (5), which is the sterile interaction eigenstate. In contrast, the DP DM is residing in a vacuum propagation eigenstate (i.e., a mass eigenstate), after a nontrivial cosmological evolution [10,64-67]. The vacuum propagation eigenstates $\left(A^{\prime}, X^{\prime}\right)$, found by diagonalizing the mass term of Eq. (2), can be related to $(A, X)$ via

\footnotetext{
${ }^{2}$ We have a different result with respect to the one quoted in Ref. [46] [see the discussion after their Eq. (9)], in which what is called "electric field" is in our case $(1-\epsilon) \mathbf{E}$. However, the final results of Ref. [46] do not depend on this expression (nor do ours, as we tune the haloscope to obtain $\epsilon \rightarrow 0$ ).
}

$\left(\begin{array}{l}A \\ X\end{array}\right)=\left(\begin{array}{cc}\cos \alpha & \sin \alpha \\ -\sin \alpha & \cos \alpha\end{array}\right)\left(\begin{array}{l}A^{\prime} \\ X^{\prime}\end{array}\right) \simeq\left(\begin{array}{cc}1 & \chi \\ -\chi & 1\end{array}\right)\left(\begin{array}{l}A^{\prime} \\ X^{\prime}\end{array}\right)$

where $\chi$ has been assumed to be small. We observe that the DM (vacuum propagation eigenstate) consists primarily of the sterile interaction eigenstate, which is the source in Eq. (12).

At this point, we can relate $\mathbf{X}$ in Eq. (12) with the DM energy density. The Hamiltonian of the DP is that of a massive photon (i.e., the Proca field),

$$
\begin{aligned}
H_{\mathrm{DP}}(t, \mathbf{x})= & \frac{1}{2}\left[\mathbf{E}_{X} \cdot \mathbf{E}_{X}+\mathbf{B}_{X} \cdot \mathbf{B}_{X}\right. \\
& \left.+m_{X}^{2}\left(X^{0} X^{0}+\mathbf{X} \cdot \mathbf{X}\right)\right],
\end{aligned}
$$

where $\mathbf{E}_{X}=\partial_{0} \mathbf{X}+\nabla X^{0}$ and $\mathbf{B}_{X}=\nabla \times \mathbf{X}$. Thus, the energy density is $[10,68]$

$$
\begin{aligned}
\rho & =\frac{1}{V} \int d^{3} \mathbf{x} H_{\mathrm{DP}}(t, \mathbf{x})=\frac{1}{V} \int d^{3} \mathbf{x} \frac{1}{2} \partial_{0} \mathbf{X} \cdot \partial_{0} \mathbf{X} \\
& =\int \frac{d^{3} \mathbf{k}}{(2 \pi)^{3}} \frac{\omega(\mathbf{k})^{2}}{2}|\mathbf{X}(\mathbf{k})|^{2},
\end{aligned}
$$

where we used the Fourier expansion in Eq. (5) and indicated the only term in $H$ whose integral is not zero.

The space average of the complex DP field is

$$
\begin{aligned}
\left\langle X_{c}^{\mu}(t)\right\rangle & =\frac{1}{V} \int d^{3} \mathbf{x} X_{c}^{\mu}(t, \mathbf{x}) \\
& =\frac{X^{\mu}(\mathbf{k}=0)}{\sqrt{V}} e^{-i m_{X} t} \equiv X_{0}^{\mu} e^{-i m_{X} t},
\end{aligned}
$$

corresponding to a plane wave with frequency $m_{X}$ whose amplitude $X_{0}^{\mu}=\left(X_{0}^{0}, \mathbf{X}_{0}\right)$ we defined as $X_{0}^{\mu}=X^{\mu}(\mathbf{k}=0) / \sqrt{V}$

Equation (15) allow us to make contact between the two different formulations of DP DM as a classical field and as particles, the latter given by the local velocity distribution of DM particles in the laboratory frame $f_{\text {lab }}(\mathbf{v})$, through

$$
\rho=\rho \int d^{3} \mathbf{v} f_{\mathrm{lab}}(\mathbf{v})
$$

Thus, taking $\mathbf{k}=m_{X} \mathbf{v}$ in Eq. (15) the DM velocity distribution is identified with [61]

$$
f_{\text {lab }}(\mathbf{v})=\frac{m_{X}^{3} \omega^{2}}{2(2 \pi)^{3} \rho}|\mathbf{X}(\mathbf{k})|^{2} .
$$

The kinetic energy of DM particles in the dark halo of our Galaxy is of $\mathcal{O}\left(10^{-6}\right) m_{X}$. Neglecting terms of this order, we can identify $\omega(\mathbf{k})^{2}=m_{\mathrm{X}}^{2}$ in Eq. (15). Then, using the inverse Fourier transform of Eq. (5) we obtain 


$$
\rho=\frac{m_{X}^{2}}{2}\left\langle\left|\mathbf{X}_{c}(t, \mathbf{x})\right|^{2}\right\rangle,
$$

where the brackets indicate the space average of the square magnitude of the DP field. Further, neglecting the DM velocity that is of $\mathcal{O}\left(10^{-3}\right)$, i.e., using

$X^{\mu}(\mathbf{k})=\frac{X^{\mu}(\mathbf{k}=0)}{V}(2 \pi)^{3} \delta^{3}(\mathbf{k})=\frac{X_{0}^{\mu}}{\sqrt{V}}(2 \pi)^{3} \delta^{3}(\mathbf{k})$

in the Fourier expansion Eq. (5) (which means approximating the local DP DM velocity distribution by $f_{\text {lab }}(\mathbf{v})=$ $\left.\left(m_{X}^{2} / 2\right) \rho\left|\mathbf{X}_{0}\right|^{2} \delta^{(3)}(\mathbf{v})\right)$, allows us to identify the DP field with the plane wave in Eq. (16). Therefore,

$\rho=\frac{m_{X}^{2}}{2}\left|\left\langle\mathbf{X}_{c}(t)\right\rangle\right|^{2}=\frac{m_{X}^{2}}{2} \frac{|\mathbf{X}(\mathbf{k}=0)|^{2}}{V}=\frac{m_{X}^{2}}{2}\left|\mathbf{X}_{0}\right|^{2}$.

This result immediately follows from Eq. (19) for a field constant in space and can be obtained directly using Eq. (20) and $\left[(2 \pi)^{3} \delta^{3}(\mathbf{k})\right]^{2}=V(2 \pi)^{3} \delta^{3}(\mathbf{k})$ in the integral of Eq. (15).

We can also make contact through the expressions we obtained for the DM energy density $\rho$ with the momentum density function $f_{X}(\mathbf{k})$ that we employ in Appendix. Writing the energy density as

$$
\rho=\int \frac{d^{3} \mathbf{k}}{(2 \pi)^{3}} \omega(\mathbf{k}) f_{X}(\mathbf{k})
$$

we can use Eq. (15) and obtain

$$
f_{X}(\mathbf{k})=\frac{\omega(\mathbf{k})}{2}|\mathbf{X}(\mathbf{k})|^{2} .
$$

Alternatively, if we neglect the DM velocity, i.e., using Eq. (20), we find

$$
f_{X}(\mathbf{k})=\frac{(2 \pi)^{3}}{2} m_{X}\left|\mathbf{X}_{0}\right|^{2} \delta^{(3)}(\mathbf{k}) .
$$

The power $P$ in a plasma is given by

$$
P=\Gamma U,
$$

where $U$ is the stored energy. The damping is given by $\Gamma=\omega / Q$, where $Q$ is the quality factor and $(\omega / Q)$ denotes the full-width-half-maximum of the signal.

For DP, the on resonance output power, when put in a suggestive form reminiscent of cavity haloscopes [10], is thus given by

$$
\begin{aligned}
P_{\text {out }} & =\kappa \Gamma \frac{1}{4} \int\left(\frac{\partial(\epsilon \omega)}{\partial \omega}|\mathbf{E}|^{2}+|\mathbf{B}|^{2}\right) d V \\
& =\kappa \chi^{2} \rho m_{X} Q V_{d} \mathcal{G}
\end{aligned}
$$

where in the first line we used the definition of energy density of the electromagnetic field within a dispersive medium [69] and in the second line we used Eq. (21) and defined the "geometric factor"

$$
\mathcal{G}=\frac{|\epsilon|^{2}}{m_{X}^{2} \chi^{2}\left|\mathbf{X}_{0}\right|^{2} V_{d}} \frac{1}{2} \int\left(\frac{\partial(\epsilon \omega)}{\partial \omega}|\mathbf{E}|^{2}+|\mathbf{B}|^{2}\right) d V,
$$

which is typically of $\mathcal{O}(1)$. Note that the $E$-field structure is the same as in the case of the axion (Ref. [52]). Here, $\rho=0.45 \mathrm{GeV} / \mathrm{cm}^{3}$ is the local DM density (the value assumed in, e.g., Ref. [70] and compatible with observations [71-73]), $V_{d}$ is the fiducial volume of the detector, and $\kappa$ is the signal coupling efficiency factor.

In Appendix, we employ the machinery of thermal field theory to directly compute the power absorbed by the detector in the "propagator approach" [55]. Our results confirm the expressions obtained with classical electrodynamics in the main text, as well as the expressions obtained with quantum field theory (with in-medium corrections), as used in, e.g., Refs. [24,29].

\section{THIN WIRE METAMATERIALS}

Recent advances in material science have devoted significant attention to metamaterials, composite materials with periodically or randomly distributed artificial microstructure with size and spacing smaller than the wavelength of interest. Their unique properties can be exploited for new physics searches.

We briefly describe wire metamaterials, which have recently been proposed for the realization of tunable plasma haloscopes [52]. As demonstrated in Ref. [74], an extended network of thin wires comprises a composite dielectric that behaves like a metal with a plasma frequency in the $\mathrm{GHz}$ range. We envision a configuration based on a system of aligned wires in the $\hat{z}$-direction. Since such a system constitutes an extremely anisotropic medium, the longitudinal and transverse modes of polarization tensor are mixed. This can be encoded through a matrix (see, e.g., Ref. [30])

$$
\mathcal{K}_{A B} \equiv e_{\mu}^{A} \Pi^{\mu \nu} e_{\nu}^{B},
$$

where the $A$ and $B$ indices run over the longitudinal and transverse polarization vectors. A nonmagnetic material has a symmetric polarization tensor and we can thus diagonalize $\mathcal{K}$. In the $z$-direction of the wires the effective dielectric constant can be described by the standard Drude model for metals [74,75], with eigenvalues

$$
\epsilon \equiv \epsilon_{z}=1-\frac{\omega_{p}^{2}}{\omega^{2}-i \omega \Gamma} \quad \text { and } \quad \epsilon_{\perp z}=1 .
$$

For a rectangular array of wires, with a wire radius $d$ and an interwire spacing in each direction perpendicular to the wires of $a$ and $b$, the plasma frequency is given by [75] 


$$
\omega_{p}^{2}=\frac{2 \pi / s^{2}}{\log \left(\frac{s}{2 \pi d}\right)+F(r)},
$$

where $s=\sqrt{a b}, r=a / b$ and

$$
F(r)=-\frac{1}{2} \log r+\sum_{n=1}^{\infty}\left(\frac{\operatorname{coth}(\pi n r)-1}{n}\right)+\frac{\pi r}{6},
$$

allowing for $\omega_{p} \sim \mathrm{GHz}$ when $s \sim \mathrm{cm}$ spacing. Since the plasma frequency is predominantly determined by the interwire spacing, this setup allows to realize a tunable plasma haloscope. The resulting electric field is thus

$$
|\mathbf{E}|=\left|\frac{\chi m_{X}}{\epsilon} \mathbf{X} \cos \theta\right|,
$$

where $\theta$ is

$$
\cos \theta=\frac{|\hat{\mathbf{z}} \cdot \mathbf{X}|}{|\mathbf{X}|}
$$

Envisioning a finite cylindrical experimental configuration, the discussion of bounded plasma solutions for the field propagation in plasma haloscopes can be found in Ref. [52].

Unlike axions, DPs can have electric fields in directions other than the $\hat{z}$-direction of wire alignment. If the boundary of the plasma is a conducting cylinder, for electric fields polarized in the nonaxial directions the device behaves like a resonant cavity. Thus, in principle, DPs can excite transverse electric modes (i.e., modes with $E_{z}=0$ ) if the mode frequency matches the DP mass. However, as the DP interacts with cavities analogously to the axion, the rate is proportional to the overlap integral of the DP wave function and the cavity mode. In the case of transverse electric modes in a cylinder, this overlap is zero [76]. Thus, we only consider $E_{z}$.

\section{EXPERIMENTAL SETUP}

We now discuss a setup configuration for DP detection with a plasma haloscope. The output power is immediately read from Eq. (26) as

$$
P_{\text {out }}=\kappa \chi^{2} \rho m_{\mathrm{X}} Q V_{d} \mathcal{G} \cos ^{2} \theta .
$$

For an unknown DP field direction at the experiment, our limits are obtained by averaging over all the possible directions, with $\left\langle\cos ^{2} \theta\right\rangle=1 / 3$. Such an average occurs when the polarization of the DP fluctuates over short timescales, so that a measurement samples many different polarizations. In the case of a fixed polarization, for a measurement longer than a day the signal will still be averaged over to give an $\mathcal{O}(1)$ number, but the exact average will depend on the angle of the DP with the Earth and the experiment's location on Earth.
For simplicity we will follow the specifications of Ref. [52]. We consider a cylindrical structure enclosing copper wires with diameter of $\sim 10 \mu \mathrm{m}$, which are readily commercially available. We take $Q=10^{2}, \kappa=0.5 .^{3}$

Unlike the axion case [52], a magnetic field is not required for DP search and hence we are not restricted by the geometric considerations related to the magnet bore size. However, such a device will still need to be cooled, which limits the volume. For a quantum limited detection at low frequencies a dilution refrigerator is required [77], which is unlikely to be possible for $V>\mathcal{O}\left(\mathrm{m}^{3}\right)$. ADMX, which has a cavity volume of $136 \mathrm{~L}$, has the largest dilution refrigerator currently used in axion detection [78]. However, one could also envision a larger, warmer system aiming at lower frequencies. Another limitation for considered volume is that heavier DPs with large de Broglie wavelength $\lambda_{\mathrm{DB}}$ will lose coherence over the experiment's dimensions, which both reduces the expected signal and increases the signal's sensitivity to the (unknown) DP velocity dispersion, similar to cavities and dielectric haloscopes [61]. Hence, each dimension of the experiment should not exceed $\mathcal{O}(10) \%$ of the DP's de Broglie wavelength.

Employing the physical dimensions for the experiment as proposed in Ref. [52], we consider a cylinder of volume $V=0.8 \mathrm{~m}^{3}$ and diameter $60 \mathrm{~cm}$. For a lower limit on detection frequency, we envision a minimum of 300 wires, which in a $60 \mathrm{~cm}$ diameter cylinder gives a wire spacing of $3 \mathrm{~cm} .{ }^{4}$ The lower limit on the number of wires stems from the requirement that the medium behaves as a plasma, which was confirmed to occur within a square array of $\gtrsim 200-400$ wires [79]. From Eq. (30), this translates into a lower limit on detection sensitivity to plasma frequency $\nu_{p}=1.5 \mathrm{GHz}$, corresponding to a DP mass of $m_{\mathrm{DP}} \simeq 6 \mu \mathrm{eV}$. These considerations also applied to Ref. [52], signifying that plasma haloscopes are more sensitive to light DM at smaller mass range than originally thought. Note that when the wavelength of light in the medium becomes larger than the experiment the power becomes suppressed [80]. For us, this translates into geometry factor approaching zero as $R / \sqrt{\epsilon_{z}} \omega \rightarrow 0$, where $R$ is the radius of the cylinder. We illustrate this suppression explicitly in the left panel of Fig. 1, where at $1.5 \mathrm{GHz} \mathcal{G}=1.7 \times 10^{-2}$-leading to a hundredfold suppression of the power. Thus, if even lower frequencies are desired, one must increase the radius of the experiment, at the possible expense of a sub Kelvin cooling system. An alternative could be to operate slightly off the

\footnotetext{
${ }^{3}$ This corresponds to a critically coupled system where the power extracted as signal is equal to other losses.

${ }^{4}$ In Ref. [52] there was an error in converting plasma frequencies to physical wire spacings, leading to the frequency associated with a given spacing being overestimated by a factor of $2 \pi$. However, as the discussed frequency limits are based on general engineering considerations and not a specific realization of the experiment, this is not very significant.
} 

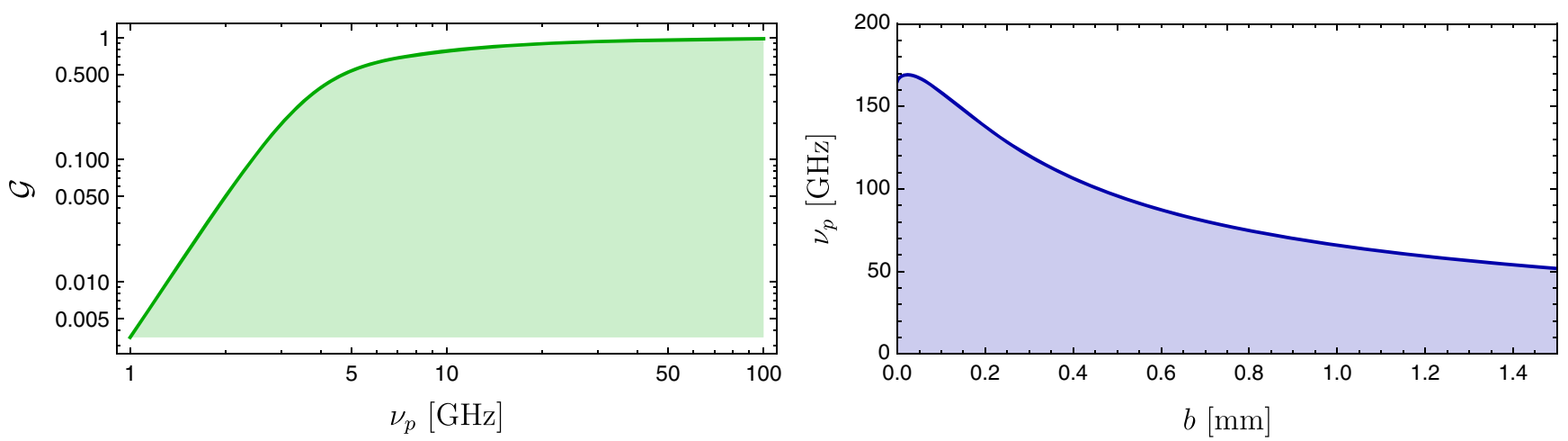

FIG. 1. Left: geometric factor $\mathcal{G}$ of Eq. (27), obtained using finite plasma field description within a wave-guide as derived in Ref. [52], describing a system of radius $30 \mathrm{~cm}$ with a quality factor $Q=100$ as a function of plasma frequency $\nu_{p}$. Right: plasma frequency $\nu_{p}=\omega_{p} /(2 \pi)$, given in Eq. (30), of a wire metamaterial configuration composed of aligned $10 \mu \mathrm{m}$ copper wires as a function of interwire spacing in orthogonal directions perpendicular to the wires, $a=1 \mathrm{~mm}$ and $b$. As the spacing $b \rightarrow 0$, the plasma frequency saturates to a maximal value. This indicates both the maximum reachable plasma frequency as well as tunability of a given system.

plasma frequency, thus modifying the wavelength to match a resonant mode of the cavity.

For considerations of a (soft) upper limit on detection frequency, we note that mechanically tuning such a device would prove very challenging for sub-mm wire spacings. However, as can be seen from Eq. (30), only one direction needs to be tuned in order to change $\omega_{p}$ [52]. Thus, one can compensate for limitations in the tuning direction by manufacturing wires to be more densely packed in an orthogonal direction. Hence, if the closest possible spacing is $a \sim 1 \mathrm{~mm}$, one could still achieve a plasma frequency of $\nu_{p}=100 \mathrm{GHz}$ with an orthogonal-direction wire spacing of $b \sim 0.5 \mathrm{~mm}$. We note that employing an arbitrarily small spacing in the direction that is not being tuned would not prove beneficial. To illustrate this, we depict in the right panel of Fig. 1 the plasma frequency of Eq. (30) as a function of spacing $b$ for a set of $10 \mu \mathrm{m}$ wires of spacing $a=1 \mathrm{~mm}$. We observe that $\omega_{p}$ is saturated as $b \rightarrow 0$ (though the formalism breaks down when the wire radius becomes comparable to the spacing). Hence, if one is restricted to mechanical tuning, the minimum spacing allowed in the tuning direction determines the maximal plasma frequency. To get to $100 \mathrm{GHz}$ would require a minimum spacing $\lesssim 1.5 \mathrm{~mm}$. However, we note that wire metamaterials can be tuned via the Josephson effect [81], which would evade such constraints.

\section{SIGNAL DETECTION}

We envision detecting the signal via an antenna coupled to the system. While the full design of such an antenna would be the subject of a detailed technical proposal, if multiple elements are needed to extract power from the full volume a summing network could be employed, as was proposed for axion detection in Ref. [82]. As the refractive index is almost purely imaginary on resonance, waves decay over a distance $1 / \sqrt{\epsilon_{z}} \omega$ in transverse (non-z) directions. Thus, systems with $R \gg 1 / \sqrt{\epsilon_{z}} \omega$ require some antenna elements to be placed inside the medium for a full readout. Under these assumptions, we can obtain the scan rate via Dicke's radiometer formula for signal-to-noise ratio $(S / N)$ (see discussion in, e.g., Ref. [83])

$$
\frac{S}{N}=\frac{P}{T_{\text {sys }}} \sqrt{\frac{\Delta t}{\Delta \nu_{\mathrm{DP}}},}
$$

where $T_{\text {sys }}$ is the system noise temperature, $\Delta \nu_{\mathrm{DP}} \simeq$ $10^{-6} \nu_{\mathrm{DP}}$ is the DP signal line width, $\Delta t$ is the measurement time that covers a frequency range $\sim Q / \omega$. Here, frequency is $\nu_{\mathrm{DP}}=\omega /(2 \pi)$, where $\omega=m_{\mathrm{DP}_{5}}$ on resonance. We consider quantum limited detection, ${ }^{5}$ taking $T_{\mathrm{sys}}=m_{\mathrm{DP}}$.

For our parameters, the power at a given frequency is

$$
\begin{aligned}
P_{\text {out }}= & 1.1 \times 10^{-22} \mathrm{~W}\left(\frac{\kappa}{0.5}\right)\left(\frac{\mathcal{G}}{1}\right)\left(\frac{\chi}{10^{-15}}\right)^{2}\left(\frac{Q}{100}\right) \\
& \times\left(\frac{V_{d}}{0.8 \mathrm{~m}^{3}}\right)\left(\frac{\nu}{10 \mathrm{GHz}}\right)\left(\frac{\rho}{0.45 \mathrm{GeV} / \mathrm{cm}^{3}}\right) .
\end{aligned}
$$

At higher masses, this equation is modified by the restriction that experimental size does not exceed $\sim 30 \%$ of $\lambda_{\mathrm{DB}}$. As we are considering a narrow aspect ratio cylindrical cavity whose height $h$ exceeds the diameter, we impose $h<0.3 \lambda_{\mathrm{DB}}$. At lower frequencies, the geometric factor $\mathcal{G}$ of Eq. (27), displayed on Fig. 1, is suppressed due to the finite size of the waveguide [52].

We can obtain a rough estimate of the experimental livetime required to scan over a region of parameter space by treating the resonance as a rectangular spectrum with a

\footnotetext{
${ }^{5}$ At low frequencies $(\lesssim 10 \mathrm{GHz})$ detection near quantum limit has been demonstrated with Josephson parametric amplifiers operating at low temperatures [77].
} 
width given by the full width half maximum $(\omega / Q)$ and height given by the half maximum $\left(P_{\text {out }} / 2\right)$. This estimate assumes that a relatively rapid frequency tuning $(\ll \Delta t)$ without disturbing the system is possible, although these effects could be included [84]. Given that a low $Q$ is assumed, each measurement is relatively long, so this is not a significant restriction on the tuning time. Thus, integrating Eq. (35), the total scanning time between frequencies $\nu_{1}$ and $\nu_{2}$ is given by

$$
t_{\text {scan }}=4 \times 10^{-6} Q \int_{\nu_{1}}^{\nu_{2}} d \nu\left(\frac{S}{N}\right)^{2}\left(\frac{T_{\text {sys }}}{P_{\text {out }}}\right)^{2} .
$$

Requiring a signal-to-noise ratio of $(S / N) \geq 3$, a kinetic mixing parameter value down to $\chi \simeq 7 \times 10^{-16}$ can be probed across our whole parameter range of $6-400 \mu \mathrm{eV}$ within $\sim 5$ years of experimental livetime.

For the above input parameters and experimental livetime of 5 years, in Fig. 2 we display the projected sensitivity for DP parameter range in green along with existing constraints. ${ }^{6}$ We note that while axion haloscope limits are often converted in the literature into limits on DPs in the vein of Ref. [10], many of the published limits have employed magnetic field as a veto on signals (e.g., Refs. $[87,88])$. Thus, any potential DP signals were rejected, signifying that one cannot directly reinterpret such studies as DP searches. In Ref. [78] such a procedure was also used, however only injected simulation candidate events were present before this was done. Hence, while it is uncertain if a DP would have been observed if there had been a candidate event, one can use the lack of candidates as a limit. In the cases which did not explicitly employ such a procedure, we assume that no veto was employed. If the analysis is done allowing for DP signals, cavity searches such as ADMX [39], HAYSTAC [89], CULTASK [40], ORGAN [41], KLASH [42] and RADES [43,44] are potentially very sensitive to DPs, particularly for $\nu \lesssim 10 \mathrm{Ghz}$. As multiple axion haloscope searches perform such vetoing, we only display published DP projected sensitivities along with existing limits, the most relevant being dielectric haloscopes like MADMAX [45,90]. With a small enough mass-gap, Dirac materials could also be sensitive in the range of our parameter space of interest [29].

To plot limits, we have assumed that the DP field direction changes relatively rapidly, so the averaged angle can be used for all experiments. As individual measurements are long, plasma and dielectric haloscopes would obtain similar results for a fixed DP direction. However, cavity haloscopes generally measure for very short periods,

\footnotetext{
${ }^{6}$ Dish antenna experiments [48], such as SHUKET [85] and Tokyo (University of Tokyo) [86], also probe this mass range. However, they are more suited for broadband searches at larger values of $\chi$ and their limits are less sensitive than the parameter range displayed.
}

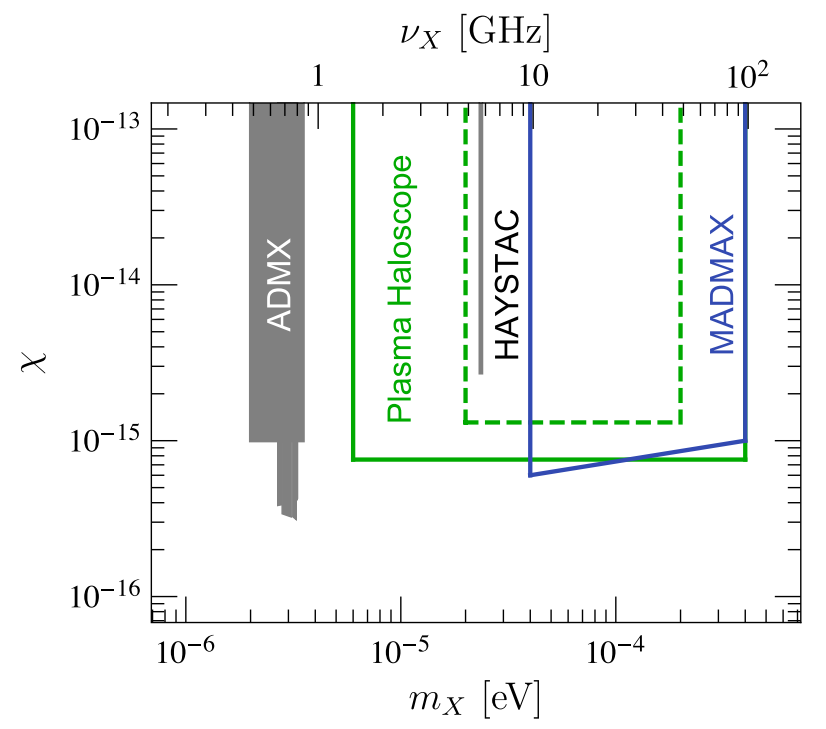

FIG. 2. Projected sensitivity reach of plasma haloscopes for DP searches assuming an $0.8 \mathrm{~m}^{3}$ volume of plasma with a quality factor $Q=100$. We assume a 5 year livetime, and display both the optimistic quantum limited (solid green) as well as $8 \mathrm{~K}$ noise temperature (dashed green) cases. Existing limits (dark gray) from haloscope cavity searches of ADMX [70,91-93] and HAYSTAC [89] are shown. We display the projected DP reach of MADMAX (blue) dielectric haloscope, which assumes a constant $8 \mathrm{~K}$ noise temperature [90].

and so would only sample a single value of $\cos ^{2} \theta$ in each measurement. Further, usually multiple measurements, presumably occurring at different times of the day, are combined to get the final limits. To get a limit on a time varying signal would require time and location information for each measurement when combined, in order to take into account signals potentially vanishing at different times of the day. As detailed timing information is usually not reported, it is non-trivial to rigorously turn existing limits into DP limits in such a scenario.

While for axions both quantum limited and constant noise temperatures were explored in Ref. [45], the limits plotted for DP in Ref. [90] assumed a more modest noise temperature of $8 \mathrm{~K}$. Thus for a direct comparison we plot also a more conservative system with $T_{\text {sys }}=8 \mathrm{~K}$ (dashed green line). We note, however, that as near quantum limited detection is currently available below $\sim 10 \mathrm{GHz}$, such a plot is somewhat pessimistic in the low mass regime. ${ }^{7}$ As shown in Fig. 2 even with conservative parameters plasma haloscopes are capable of searching a large fraction of the DP

\footnotetext{
${ }^{7}$ Recently an alternatively designed dielectric haloscope, DALI [94], was proposed. However, Ref. [94] neglected the quantum limit of linear amplification. HAYSTAC is exploring using squeezed states with Josephson parametric amplifiers at lower frequencies to evade the quantum limit [95], however Ref. [94] assumed linear amplifiers would be used, desiring commercially available technology.
} 
parameter space, being complementary to dielectric haloscopes like MADMAX. In particular, if both types of experiments use mechanical tuning, the smaller spacings between elements in plasma haloscopes for a given frequency make it more suitable for somewhat lower frequencies.

\section{SIGNAL MODULATION}

Due to Earth's motion with respect to the Galaxy, shown schematically in Fig. 3, there is a time modulation of a DM signal, since the DM distribution is constant in time in the Galactic rest frame during the duration of any experiment (although the local DM distribution at the experiment may change if it enters and/or exists a small enough DM lump or stream, as we comment below). Equation (18), where $\mathbf{v}$ is the velocity of a DM particle in the detector's rest frame, relates the classical field and the particle formulations of DP DM. Aside from the velocity and location of the detector, the orientation of the direction of the wires $\hat{\mathbf{z}}$ in Eq. (33) also changes periodically in the Galactic rest frame.

This leads to three distinct time modulation effects (see, e.g., [83]): (1) the periodic change of the detector speed with respect to the Galaxy, (2) a possible change in DM density during data taking, and (3), the daily change in orientation of the detector with respect to the Galaxy (due to Earth's rotation about itself). This last effect occurs only for DP, but not axions, in some DM generation models and makes use of the strong directionality of the detector we consider.

The time modulation effects due to the periodic change in the velocity of the detector in the Galactic rest frame are very different when the DM particle is absorbed compared to when it scatters off and deposits only a portion of its kinetic energy within the detector.

When the DM particle is absorbed, the energy deposited in the detector is the particle mass $m_{X}$ plus its kinetic energy of $\mathcal{O}\left(10^{-6}\right) m_{X}$, since the characteristic DM speed is $\mathcal{O}\left(10^{-3}\right) c$. The DM signal obtains a finite width due to the spread of the kinetic energy. The peak frequency of the DM line is at $\omega_{\text {peak }}=m_{X}\left(1+v_{\text {Sun }}^{2} / 2\right)$, where $v_{\text {Sun }}$, the speed of the Sun with respect to the Galaxy, is about $240 \mathrm{~km} / \mathrm{s}$ (see e.g., [72]



FIG. 3. Out of scale diagram of Earth's motion with respect to the Galaxy, due to both the orbital and the Solar System motions. We also show the direction of the north galactic pole $\mathrm{N}_{\mathrm{gal}}$ and the north ecliptic pole $\mathrm{N}_{\mathrm{ecl}}$. for a discussion of the uncertainties in this speed). The orbital motion of Earth, with speed close to $30 \mathrm{~km} / \mathrm{s}$, produces an annual modulation of amplitude $\Delta v$ of the detector's speed with respect to the Galaxy, and thus a periodic shift $\left(v_{\text {Sun }} \Delta v\right) \omega_{\text {peak }}$ of $\mathcal{O}\left(10^{-7}\right) \omega_{\text {peak }}$ in the DM line peak frequency. Hence, detecting this modulation would require an experimental energy resolution of $\mathcal{O}\left(10^{-7}\right) m_{X}$.

The existence of a escape speed $v_{\text {esc }}$ of about $550 \mathrm{~km} / \mathrm{s}$ from the Galaxy at Earth's location introduces a high frequency cutoff $\omega_{\max }=m_{X}\left(1+\left(v_{\text {esc }}+v_{\text {Sun }}\right)^{2} / 2\right)$ of the DM line. The escape speed has been measured by the RAVE and GAIA surveys, with values between $480 \mathrm{~km} / \mathrm{s}$ and $640 \mathrm{~km} / \mathrm{s}$ [96-98] with respect to the Galaxy (considering the $90 \%$ C.L. intervals of each measurement). The cutoff could be difficult to measure due to the fast decrease of the DM speed distribution with increasing speed, but it is also annually modulated with an amplitude $\left[\left(v_{\text {esc }}+v_{\text {Sun }}\right) \Delta v\right] \omega_{\max }$ of $\mathcal{O}\left(10^{-6}\right) \omega_{\max }$.

The daily rotation of Earth around itself, whose surface speed at the equator is $\mathcal{O}\left(10^{-6}\right) c$, would induce an even smaller daily modulation of the peak frequency $\omega_{\text {peak }}$ (and also of $\left.\omega_{\max }\right)$. This would consequently require an even finer energy resolution of $\mathcal{O}\left(10^{-9}\right) m_{X}$.

The extremely good energy resolution required to detect the annual modulation of DM line peak, and possibly its daily modulation, could be achievable with a long enough measurement time once a signal has been observed. The potential detection of the time modulation of the peak frequency, as well as other features of the DM line shape, due e.g., to Sun's gravitational lensing, DM streams, or a dark disk, have been studied at length, primarily in the context of axion detection, see, e.g., [61,83,99-103].

A seemingly erratic time modulation in the amplitude of the signal could occur due to the existence of pervasive very dense and small (relative to the size of the Solar System) clumps of the DM. These clumps are predicted if DP DM is produced due to fluctuations of the vector field during inflation [14], as described in Ref. [83], e.g., if the experiment passes through a clump about every day and spends several seconds within it.

An important daily modulation of the DP signal could arise due to the strong dependence of the output power of Eq. (34) on the orientation of the DP vector field $\mathbf{X}$ with respect to the direction of the wires in the experiment,

$$
P_{\text {out }}=P_{\text {out }}^{\mathrm{MAX}} \cos ^{2} \theta .
$$

A daily modulation would only arise if the direction of the DP field is fixed in a large enough region of the Galaxy near Earth, such that the experiment spends at least several days within it. This could be possible if the DP DM is produced through a misalignment mechanism after inflation, depending on details of structure formation in the Universe (see, e.g., Ref. [10]). The possibility of observing a directional modulation has been studied for other detectors for DP 
detection (see, e.g., Ref. [68]) and also for axion detection (see, e.g., Ref. [61]). In the most favorable case in which our detector is located at $45^{\circ}$ latitude and the direction of $\mathbf{X}$ coincides with the direction of the wires, assumed to be in vertical position in the detector, at one time of the day (so that $\cos \theta=1$ at this instant) the daily modulation would be maximal. The output power would be maximal $P_{\text {out }}=$ $P_{\mathrm{out}}^{\mathrm{MAX}}$ at one time of the day, and 12 hours later (when the azimuth at the location of the experiment would be at $90^{\circ}$ with respect to the original position, thus $\cos \theta=0$ ) the power would be $P_{\text {out }}=0$. Thus, in this most favorable case, the daily modulation of the output power would be $100 \%$. Even just splitting the daily observation into two equal time interval bins in this most favorable case, the total signal in the half day centered at the instant of maximum power would contain $92.4 \%$ of the daily signal (and the other half just the remaining $7.6 \%$ ), which would make for a very distinguishable daily modulation. Instead, in the worst case scenario, if the $\mathbf{X}$ direction coincides with Earth's rotation axis, $\cos \theta$ would be a constant (and related to the latitude of the experiment, always assuming vertical wires) and the daily modulation would be zero.

The observation of this directional daily modulation of the signal would not only allow to reconstruct the direction of the field $\mathbf{X}$ with respect to the detector, and thus with respect to the Galaxy (any direction in the experiment frame can be easily expressed in the Galactic rest frame and vice versa-see, e.g., Appendix A of Ref. [104], and Ref. [105]), but could also point toward the DP DM production through the misalignment mechanism.

If the DP DM is instead produced through quantum fluctuations of the vector field during inflation, the direction of the field could change many times during the duration of the experiment, and even during a day. As already mentioned in Ref. [83], in this latter case an optimal setup using simultaneously multiple experiments aligned in different directions would be require to determine the instantaneous direction of $\mathbf{X}$.

If a signal is observed, the observation of a daily or annual modulation of the signal would be crucial to clearly identify the signal as due to DP DM.

\section{CONCLUSIONS}

In this work we have shown that recently proposed plasma haloscopes are particularly well suited for DP DM searches. Plasma haloscopes take advantage of in-medium effects, which suppress the signal in conventional searches. Using description based on thermal field theory as well as classical electrodynamics, we have confirmed the DP absorption rate at the experiment. By employing metamaterials, the plasma frequency in plasma haloscopes can be tuned to match the DP mass, which allows to competitively probe the region of the parameter space with DP masses of 6-400 $\mu \mathrm{eV}$. Once detected, analysis of signal modulation will allow for a definitive test that DM has been observed and could shed light on the production mechanism.

\section{ACKNOWLEDGMENTS}

We thank Francesco Capozzi, Jòn Gudmundsson, Matthew Lawson, Georg Raffelt, Karl Van Bibber, and Frank Wilczek for helpful discussions. A. M. thanks Thierry Grenet for pointing out the factor of $2 \pi$ error in Ref. [52]. A. M. is supported by the European Research Council under Grant No. 742104 and is supported in part by the research environment grant "Detecting Axion Dark Matter In The Sky And In The Lab (AxionDM)" funded by the Swedish Research Council (VR) under No. Dnr 201902337. The work of G. G., V. T., and E. V. was supported by the U.S. Department of Energy (DOE) Grant No. DESC0009937.

\section{APPENDIX: ABSORPTION RATE FROM THERMAL FIELD THEORY}

Here we employ thermal field theory to obtain the DP DM absorption rate, following the discussion of Refs. [55,106]. The emission and absorption rates of a boson by a medium is related to the self-energy of the particle in the medium itself as [107,108]

$$
\operatorname{Im} \Pi=-\omega \Gamma,
$$

where $\Gamma=\Gamma_{\mathrm{abs}}-\Gamma_{\text {prod }}$ is the rate with which the considered particle distributions approach thermal equilibrium. Thus, the absorption rate is obtained by calculating the inmedium DP self-energy. This statement corresponds to the optical theorem in the framework of thermal field theory.

We need to generalize the approach used for dark photon production by an electromagnetic plasma as in Ref. [55] to the case in which a non-thermal population of cold DM is absorbed by a detector. Let us use the momentum distribution $f_{X}(\mathbf{k})$ so that the DP number density $n$ is

$$
n=\int \frac{d^{3} \mathbf{k}}{(2 \pi)^{3}} f_{X}
$$

For a boson with absorption rate $\Gamma_{\mathrm{abs}}$ and production rate $\Gamma_{\text {prod }}$ one finds [107]

$$
\frac{\partial f}{\partial t}=-f \Gamma_{\mathrm{abs}}+(1+f) \Gamma_{\text {prod }} .
$$

We consider that only $A$ and $X$ interconvert in the medium so that the production rate of one is equal to the absorption rate of the other. Since the plasma haloscope is at cryogenic temperatures there is no separate population of photons in the medium. For the DP masses considered here the occupation number is large, $f_{X} \gg 1$. Hence, the $\mathrm{DP}$ and photon evolve respectively as 


$$
\begin{aligned}
& \frac{\partial f_{X}}{\partial t} \simeq-f_{X} \Gamma^{X} \\
& \frac{\partial f_{A}}{\partial t}=-f_{A} \Gamma_{\text {prod }}^{X}+\left(1+f_{A}\right) \Gamma_{\text {abs }}^{X},
\end{aligned}
$$

where $\Gamma^{X}=\Gamma_{\text {abs }}^{X}-\Gamma_{\text {prod }}^{X}$. Here, we are assuming that the passage from vacuum to the haloscope is strongly nonadiabatic, so that the components in the basis of interaction eigenstates of the vacuum propagation eigenstate constituting the DM are conserved after the interface. This means that the active component is small right after passing through the medium interface (as it is proportional to $\chi$, not $\chi / \epsilon)$, while the sterile component is large. This is analogous to the case of neutrinos oscillating in a matter potential in the "slab approximation" $[109,110]$.

Using $\partial f_{A} / \partial t=-\partial f_{X} / \partial t$, one has

$$
\Gamma_{\mathrm{abs}}^{X} \simeq\left(f_{X}-f_{A}\right) \Gamma^{X} \simeq-f_{X} \frac{\operatorname{Im} \Pi_{X}}{\omega},
$$

where in the last approximation we have assumed that $f_{X} \gg f_{A}$ and used Eq. (A1).

At lowest order the DP self-energy, shown in Fig. 4, is given by [55]

$$
\Pi_{X}^{L, T}=m_{X}^{2}+m_{X}^{2} \chi \frac{1}{K^{2}-\Pi_{L, T}(K)} m_{X}^{2} \chi,
$$

where $K=(\omega, \mathbf{k})$ is the four-momentum of the external DP and $\Pi_{L, T}$ are the self-energy of the longitudinal and transverse plasmons respectively (we drop $A$ in the notation). Both longitudinal and transverse excitations can be produced by the DP population, which can be absorbed both when with longitudinal and transverse polarization.

From Eq. (A6), it is seen that we need the plasmon selfenergy to obtain the DP absorption rate. The real part, which modifies the dispersion relations of photons in the medium,

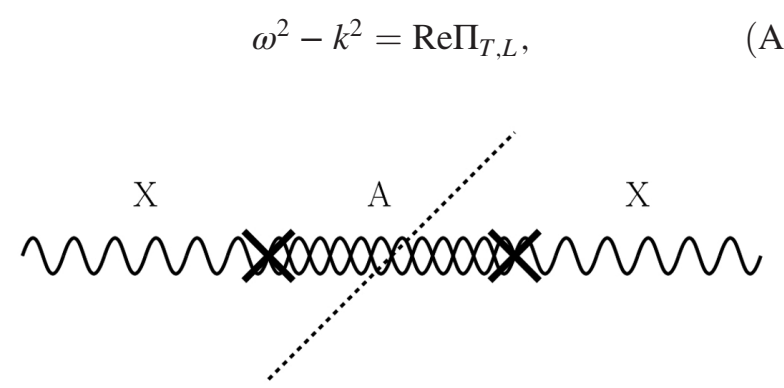

FIG. 4. Feynman diagram representing the DP (single line) self energy contribution due to the mixing with the plasmon (double line), through the vertex $\chi m_{X}^{2}$ (crosses). The plasmon propagator is cut by the diagonal dashed line to indicate that its frequency is taken to be equal to the DP mass as its momentum goes to zero, i.e., $\omega=m_{X}=\omega_{p}$. in the nonrelativistic approximation is given by [55]

$$
\begin{aligned}
& \operatorname{Re} \Pi_{T}=\omega_{p}^{2}, \\
& \operatorname{Re} \Pi_{L}=\frac{K^{2}}{\omega^{2}} \omega_{p}^{2},
\end{aligned}
$$

where $\omega_{p}$ is the plasma frequency. These are Eqs. (6.32) and (6.38) of Ref. [53], considering that they are written for the case in which $\operatorname{Im} \Pi_{T, L}=0$. We see that the dispersion relation for the transverse plasmon gives $\omega^{2}-k^{2}=\omega_{p}^{2}$, with the usual interpretation of transverse excitations as particles with mass $\omega_{p}$. The latter is given in a system of wires by Eq. (30). The longitudinal plasmon, on the other hand, has a peculiar dispersion relation, so that in the nonrelativistic limit $\omega$ is independent from $k$. The imaginary part of the photon self-energy is related to the rate $\Gamma$ by which plasmons thermalize in the medium-i.e., the damping in the classical description.

When treating longitudinal plasmons one defines the vertex renormalization constant $Z_{L}$ [54]

$$
K^{2}=Z_{L}^{-1} \omega^{2}
$$

relevant for the coupling of external photons or plasmons to electrons in the medium. We then interpret

$$
\Gamma_{L}=-Z_{L} \operatorname{Im} \Pi_{L} / \omega
$$

Enforcing the on-shell condition $K^{2}=m_{X}^{2}$, the resulting absorption rate of DPs is

$$
\begin{aligned}
\Gamma_{\mathrm{abs}}^{X}= & f_{X}^{L} m_{X}^{2} \omega^{2} \frac{\chi^{2} \Gamma_{L}}{\left(\omega^{2}-\omega_{p}^{2}\right)^{2}+\left(\omega \Gamma_{L}\right)^{2}} \\
& +f_{X}^{T} m_{X}^{4} \frac{\chi^{2} \Gamma_{T}}{\left(m_{X}^{2}-\omega_{p}^{2}\right)^{2}+\left(\omega \Gamma_{T}\right)^{2}},
\end{aligned}
$$

where we have defined the longitudinal and transverse DP populations $\left(f_{X}^{L}\right.$ and $f_{X}^{T}$, respectively, and $f_{X}^{L}+f_{X}^{T}=f_{X}$ ), and we have used Eq. (A1) to define $\Gamma_{T}=-\operatorname{Im} \Pi_{T} / \omega$. This expression closely resembles the production rate of $X$ bosons obtained in Ref. [111]. Moreover, interpreting

$$
\chi_{\mathrm{eff}}^{2}=\chi^{2} \frac{m_{X}^{4}}{\left(m_{X}^{2}-\omega_{p}^{2}\right)^{2}+\left(\omega \Gamma_{T}\right)^{2}}
$$

as an effective coupling, we find the same expression as Eq. (4.4) of Ref. [29] (see also Refs. [24,30]).

The longitudinal and transverse photons are indistinguishable in the zero momentum limit, $\Gamma_{T}=\Gamma_{L} \equiv \Gamma$, so that for nonrelativistic DPs on resonance $\left(\omega=m_{X}=\omega_{p}\right)$ the number absorption rate per unit volume is 


$$
\Gamma_{\mathrm{abs}}^{X}=f_{X} \frac{\chi^{2} m_{X}^{2}}{\Gamma}
$$

As long as the dark matter distribution is narrower than the line width of the resonance, the DP momentum distribution can be written as

$$
f_{X}=n(2 \pi)^{3} \delta^{3}(\mathbf{k})
$$

where $n=\rho / m_{X}$ is the DM number density, which coincides with Eqs. (24) and (21). Thus, the power absorbed in a homogeneous and isotropic detector with volume $V_{d}$ is given by

$$
P=V_{d} \int \frac{d^{3} \mathbf{k}}{(2 \pi)^{3}} \omega \Gamma_{\mathrm{abs}}^{X}=\chi^{2} \rho m_{X}^{2} \frac{Q}{m_{X}} V_{d}
$$

where $Q=\omega / \Gamma$ is the quality factor and $\rho$ is the local DM density. Equation (A15) is equivalent to Eq. (26) in the main text

$$
P_{\text {out }}=\kappa P,
$$

in the limit where boundary conditions are negligible so that the "geometric factor" goes to unity.
[1] G. B. Gelmini, The hunt for dark matter, in TASI: Journeys Through the Precision Frontier: Amplitudes for Colliders (2015), pp. 559-616, https://inspirehep.net/conferences/ 1276758.

[2] J. Jaeckel and A. Ringwald, The low-energy frontier of particle physics, Annu. Rev. Nucl. Part. Sci. 60, 405 (2010).

[3] M. Pospelov, A. Ritz, and M. B. Voloshin, Bosonic superWIMPs as keV-scale dark matter, Phys. Rev. D 78, 115012 (2008).

[4] H. An, M. Pospelov, and J. Pradler, Dark Matter Detectors as Dark Photon Helioscopes, Phys. Rev. Lett. 111, 041302 (2013).

[5] M. Goodsell, J. Jaeckel, J. Redondo, and A. Ringwald, Naturally light hidden photons in LARGE volume string compactifications, J. High Energy Phys. 11 (2009) 027.

[6] J. Preskill, M. B. Wise, and F. Wilczek, Cosmology of the invisible axion, Phys. Lett. 120B, 127 (1983).

[7] L. Abbott and P. Sikivie, A cosmological bound on the invisible axion, Phys. Lett. 120B, 133 (1983).

[8] M. Dine and W. Fischler, The not so harmless axion, Phys. Lett. 120B, 137 (1983).

[9] A. E. Nelson and J. Scholtz, Dark light, dark matter and the misalignment mechanism, Phys. Rev. D 84, 103501 (2011).

[10] P. Arias, D. Cadamuro, M. Goodsell, J. Jaeckel, J. Redondo, and A. Ringwald, WISPy cold dark matter, J. Cosmol. Astropart. Phys. 06 (2012) 013.

[11] G. Alonso-Álvarez, T. Hugle, and J. Jaeckel, Misalignment \& Co.: (Pseudo-)scalar and vector dark matter with curvature couplings, J. Cosmol. Astropart. Phys. 02 (2020) 014.

[12] K. Nakayama, Vector coherent oscillation dark matter, J. Cosmol. Astropart. Phys. 10 (2019) 019.

[13] K. Nakayama, Constraint on vector coherent oscillation dark matter with kinetic function, arXiv:2004.10036.

[14] P. W. Graham, J. Mardon, and S. Rajendran, Vector dark matter from inflationary fluctuations, Phys. Rev. D 93, 103520 (2016).

[15] Y. Nakai, R. Namba, and Z. Wang, Light dark photon dark matter from inflation, arXiv:2004.10743.
[16] J. A. Dror, K. Harigaya, and V. Narayan, Parametric resonance production of ultralight vector dark matter, Phys. Rev. D 99, 035036 (2019).

[17] P. Agrawal, N. Kitajima, M. Reece, T. Sekiguchi, and F. Takahashi, Relic abundance of dark photon dark matter, Phys. Lett. B 801, 135136 (2020).

[18] R. T. Co, A. Pierce, Z. Zhang, and Y. Zhao, Dark photon dark matter produced by axion oscillations, Phys. Rev. D 99, 075002 (2019).

[19] M. Bastero-Gil, J. Santiago, L. Ubaldi, and R. VegaMorales, Vector dark matter production at the end of inflation, J. Cosmol. Astropart. Phys. 04 (2019) 015.

[20] A. J. Long and L.-T. Wang, Dark photon dark matter from a network of cosmic strings, Phys. Rev. D 99, 063529 (2019).

[21] E. Aprile et al. (XENON Collaboration), First Dark Matter Search Results from the XENON1T Experiment, Phys. Rev. Lett. 119, 181301 (2017).

[22] E. Aprile et al. (XENON Collaboration), Dark Matter Search Results from a One Ton-Year Exposure of XENON1T, Phys. Rev. Lett. 121, 111302 (2018).

[23] D. Akerib et al. (LUX Collaboration), Results from a Search for Dark Matter in the Complete LUX Exposure, Phys. Rev. Lett. 118, 021303 (2017).

[24] H. An, M. Pospelov, J. Pradler, and A. Ritz, Direct detection constraints on dark photon dark matter, Phys. Lett. B 747, 331 (2015).

[25] I. M. Bloch, R. Essig, K. Tobioka, T. Volansky, and T.-T. Yu, Searching for dark absorption with direct detection experiments, J. High Energy Phys. 06 (2017) 087.

[26] Y. Hochberg, Y. Zhao, and K. M. Zurek, Superconducting Detectors for Superlight Dark Matter, Phys. Rev. Lett. 116, 011301 (2016).

[27] Y. Hochberg, M. Pyle, Y. Zhao, and K. M. Zurek, Detecting superlight dark matter with Fermi-degenerate materials, J. High Energy Phys. 08 (2016) 057.

[28] Y. Hochberg, Y. Kahn, M. Lisanti, C. G. Tully, and K. M. Zurek, Directional detection of dark matter with twodimensional targets, Phys. Lett. B 772, 239 (2017). 
[29] Y. Hochberg, Y. Kahn, M. Lisanti, K. M. Zurek, A. G. Grushin, R. Ilan, S. M. Griffin, Z.-F. Liu, S. F. Weber, and J.B. Neaton, Detection of sub-MeV dark matter with three-dimensional Dirac materials, Phys. Rev. D 97, 015004 (2018).

[30] A. Coskuner, A. Mitridate, A. Olivares, and K. M. Zurek, Directional dark matter detection in anisotropic dirac materials, arXiv:1909.09170.

[31] S. Knapen, T. Lin, and K. M. Zurek, Light dark matter in superfluid helium: Detection with multi-excitation production, Phys. Rev. D 95, 056019 (2017).

[32] K. Schutz and K. M. Zurek, Detectability of Light Dark Matter with Superfluid Helium, Phys. Rev. Lett. 117, 121302 (2016).

[33] A. Caputo, A. Esposito, and A. D. Polosa, Sub-MeV dark matter and the Goldstone modes of superfluid helium, Phys. Rev. D 100, 116007 (2019).

[34] F. Acanfora, A. Esposito, and A. D. Polosa, Sub-GeV dark matter in superfluid He-4: An effective theory approach, Eur. Phys. J. C 79, 549 (2019).

[35] W. Guo and D. N. McKinsey, Concept for a dark matter detector using liquid helium-4, Phys. Rev. D 87, 115001 (2013).

[36] S. Knapen, T. Lin, M. Pyle, and K. M. Zurek, Detection of light dark matter with optical phonons in polar materials, Phys. Lett. B 785, 386 (2018).

[37] I. G. Irastorza and J. Redondo, New experimental approaches in the search for axion-like particles, Prog. Part. Nucl. Phys. 102, 89 (2018).

[38] P. Sikivie, Experimental Tests of the Invisible Axion, Phys. Rev. Lett. 51, 1415 (1983); Erratum, Phys. Rev. Lett. 52, 695 (1984)

[39] G. Rybka (ADMX Collaboration), Direct detection searches for axion dark matter, Phys. Dark Universe 4, 14 (2014).

[40] W. Chung, Launching axion experiment at CAPP/IBS in Korea, in Proceedings, 12th Patras Workshop on Axions, WIMPs and WISPs: Jeju Island, South Korea, 2016, DESY: Hamburg, Germany (2017), pp. 30-34, http:// bib-pubdb1.desy.de/record/395473.

[41] M. Goryachev, B. T. Mcallister, and M. E. Tobar, Axion detection with negatively coupled cavity arrays, Phys. Lett. A 382, 2199 (2018).

[42] D. Alesini, D. Babusci, D. Di Gioacchino, C. Gatti, G. Lamanna, and C. Ligi, The KLASH proposal, arXiv: 1707.06010.

[43] A. A. Melcón et al., Axion searches with microwave filters: The RADES project, J. Cosmol. Astropart. Phys. 05 (2018) 040.

[44] A. A. Melcón et al., Scalable haloscopes for axion dark matter detection in the $30 \mu \mathrm{eV}$ range with RADES, arXiv: 2002.07639.

[45] A. Caldwell, G. Dvali, B. Majorovits, A. Millar, G. Raffelt, J. Redondo, O. Reimann, F. Simon, and F. Steffen (MADMAX Working Group Collaboration), Dielectric Haloscopes: A New Way to Detect Axion Dark Matter, Phys. Rev. Lett. 118, 091801 (2017).

[46] M. Baryakhtar, J. Huang, and R. Lasenby, Axion and hidden photon dark matter detection with multilayer optical haloscopes, Phys. Rev. D 98, 035006 (2018).
[47] J. Jaeckel and J. Redondo, An antenna for directional detection of WISPy dark matter, J. Cosmol. Astropart. Phys. 11 (2013) 016.

[48] D. Horns, J. Jaeckel, A. Lindner, A. Lobanov, J. Redondo, and A. Ringwald, Searching for WISPy cold dark matter with a dish antenna, J. Cosmol. Astropart. Phys. 04 (2013) 016.

[49] J. Suzuki, T. Horie, Y. Inoue, and M. Minowa, Experimental Search for Hidden Photon CDM in the eV mass range with a Dish Antenna, J. Cosmol. Astropart. Phys. 09 (2015) 042.

[50] D. Veberič et al. (FUNK Experiment Collaboration), Search for hidden-photon dark matter with the FUNK experiment, Proc. Sci, ICRC2017 (2018) 880 [arXiv: 1711.02958].

[51] http://www.iexp.uni-hamburg.de/groups/astroparticle/brass/ brassweb.htm.

[52] M. Lawson, A. J. Millar, M. Pancaldi, E. Vitagliano, and F. Wilczek, Tunable Axion Plasma Haloscopes, Phys. Rev. Lett. 123, 141802 (2019).

[53] G. G. Raffelt, Stars as Laboratories for Fundamental Physics (University of Chicago Press, Chicago, USA, 1996).

[54] H. An, M. Pospelov, and J. Pradler, New stellar constraints on dark photons, Phys. Lett. B 725, 190 (2013).

[55] J. Redondo and G. Raffelt, Solar constraints on hidden photons re-visited, J. Cosmol. Astropart. Phys. 08 (2013) 034.

[56] C. Dvorkin, T. Lin, and K. Schutz, Making dark matter out of light: Freeze-in from plasma effects, Phys. Rev. D 99, 115009 (2019).

[57] N. Kurinsky, D. Baxter, Y. Kahn, and G. Krnjaic, A dark matter interpretation of excesses in multiple direct detection experiments, arXiv:2002.06937.

[58] J. Kozaczuk and T. Lin, Plasmon production from dark matter scattering, Phys. Rev. D 101, 123012 (2020).

[59] B. Holdom, Two U(1)'s and epsilon charge shifts, Phys. Lett. 166B, 196 (1986).

[60] M. Fabbrichesi, E. Gabrielli, and G. Lanfranchi, The dark photon, arXiv:2005.01515.

[61] S. Knirck, A. J. Millar, C. A. O'Hare, J. Redondo, and F. D. Steffen, Directional axion detection, J. Cosmol. Astropart. Phys. 11 (2018) 051.

[62] M. Haft, G. Raffelt, and A. Weiss, Standard and nonstandard plasma neutrino emission revisited, Astrophys. J. 425, 222 (1994); 438, 1017(E) (1995).

[63] H. A. Weldon, Covariant calculations at finite temperature: The relativistic plasma, Phys. Rev. D 26, 1394 (1982).

[64] S. D. McDermott and S. J. Witte, Cosmological evolution of light dark photon dark matter, Phys. Rev. D 101, 063030 (2020).

[65] S. J. Witte, S. Rosauro-Alcaraz, S. D. McDermott, and V. Poulin, Dark photon dark matter in the presence of inhomogeneous structure, J. High Energy Phys. 06 (2020) 132.

[66] A. Caputo, H. Liu, S. Mishra-Sharma, and J. T. Ruderman, Dark photon oscillations in our inhomogeneous Universe, arXiv:2002.05165. 
[67] S. Dubovsky and G. Hernández-Chifflet, Heating up the galaxy with hidden photons, J. Cosmol. Astropart. Phys. 12 (2015) 054.

[68] J. Jaeckel and S. Knirck, Directional resolution of dish antenna experiments to search for WISPy dark matter, J. Cosmol. Astropart. Phys. 01 (2016) 005.

[69] L. D. Landau, J. Bell, M. Kearsley, L. Pitaevskii, E. Lifshitz, and J. Sykes, Electrodynamics of Continuous Media (Elsevier, 2013), Vol. 8, ISBN 9781483293752.

[70] S. Asztalos et al. (ADMX Collaboration), A SQUIDBased Microwave Cavity Search for Dark-Matter Axions, Phys. Rev. Lett. 104, 041301 (2010).

[71] P.F. de Salas, Dark matter local density determination based on recent observations, J. Phys. Conf. Ser. 1468, 012020 (2020).

[72] M. Benito, A. Cuoco, and F. Iocco, Handling the uncertainties in the galactic dark matter distribution for particle dark matter searches, J. Cosmol. Astropart. Phys. 03 (2019) 033.

[73] J. Read, The local dark matter density, J. Phys. G 41, 063101 (2014).

[74] J. B. Pendry, A. J. Holden, D. J. Robbins, and W. J. Stewart, Low frequency plasmons in thin-wire structures, J. Phys. Condens. Matter 10, 4785 (1998).

[75] P. A. Belov, R. Marqués, S. I. Maslovski, I. S. Nefedov, M. Silveirinha, C. R. Simovski, and S. A. Tretyakov, Strong spatial dispersion in wire media in the very large wavelength limit, Phys. Rev. B 67, 113103 (2003).

[76] I. Stern, A. Chisholm, J. Hoskins, P. Sikivie, N. Sullivan, D. Tanner, G. Carosi, and K. van Bibber, Cavity design for high-frequency axion dark matter detectors, Rev. Sci. Instrum. 86, 123305 (2015).

[77] L. Zhong et al. (HAYSTAC Collaboration), Results from phase 1 of the HAYSTAC microwave cavity axion experiment, Phys. Rev. D 97, 092001 (2018).

[78] T. Braine et al. (ADMX Collaboration), Extended Search for the Invisible Axion with the Axion Dark Matter Experiment, Phys. Rev. Lett. 124, 101303 (2020).

[79] P. Gay-Balmaz, C. Maccio, and O. J. F. Martin, Microwire arrays with plasmonic response at microwave frequencies, Appl. Phys. Lett. 81, 2896 (2002).

[80] J. Ouellet and Z. Bogorad, Solutions to axion electrodynamics in various geometries, Phys. Rev. D 99, 055010 (2019).

[81] M. Trepanier, D. Zhang, L. V. Filippenko, V. P. Koshelets, and S.M. Anlage, Tunable superconducting Josephson dielectric metamaterial, AIP Adv. 9, 105320 (2019).

[82] C.-L. Kuo, Large-volume centimeter-wave cavities for axion searches, J. Cosmol. Astropart. Phys. 06 (2020) 010.

[83] S. Chaudhuri, K. Irwin, P. W. Graham, and J. Mardon, Fundamental limits of electromagnetic axion and hiddenphoton dark matter searches: Part I-The quantum limit, arXiv: 1803.01627.

[84] A. J. Millar, G. G. Raffelt, J. Redondo, and F. D. Steffen, Dielectric haloscopes to search for axion dark matter: Theoretical foundations, J. Cosmol. Astropart. Phys. 01 (2017) 061.

[85] P. Brun, L. Chevalier, and C. Flouzat, Direct Searches for Hidden-Photon Dark Matter with the SHUKET Experiment, Phys. Rev. Lett. 122, 201801 (2019).
[86] J. Suzuki, Y. Inoue, T. Horie, and M. Minowa, Hidden photon CDM search at Tokyo, in 11th Patras Workshop on Axions, WIMPs and WISPS (2015), pp. 145-148, https:// inspirehep.net/conferences/1332843.

[87] W. Wuensch, S. De Panfilis-Wuensch, Y. Semertzidis, J. Rogers, A. Melissinos, H. Halama, B. Moskowitz, A. Prodell, W. Fowler, and F. Nezrick, Results of a laboratory search for cosmic axions and other weakly coupled light particles, Phys. Rev. D 40, 3153 (1989).

[88] S. De Panfilis, A. Melissinos, B. Moskowitz, J. Rogers, Y. Semertzidis, W. Wuensch, H. Halama, A. Prodell, W. Fowler, and F. Nezrick, Limits on the Abundance and Coupling of Cosmic Axions at $4.5 \mu \mathrm{eV}<m_{a}<5.0 \mu \mathrm{eV}$, Phys. Rev. Lett. 59, 839 (1987).

[89] B. Brubaker et al., First Results from a Microwave Cavity Axion Search at $24 \mu \mathrm{eV}$, Phys. Rev. Lett. 118, 061302 (2017).

[90] P. Brun et al. (MADMAX Collaboration), A new experimental approach to probe QCD axion dark matter in the mass range above $40 \mu \mathrm{eV}$, Eur. Phys. J. C 79, 186 (2019).

[91] S. J. Asztalos et al. (ADMX Collaboration), Experimental constraints on the axion dark matter halo density, Astrophys. J. Lett. 571, L27 (2002).

[92] S. J. Asztalos, R. F. Bradley, L. Duffy, C. Hagmann, D. Kinion, D. M. Moltz, L. J. Rosenberg, P. Sikivie, W. Stoeffl, N. S. Sullivan, D. B. Tanner, K. van Bibber, and D. B. Yu, Improved rf cavity search for halo axions, Phys. Rev. D 69, 011101 (2004).

[93] N. Du et al. (ADMX Collaboration), A Search for Invisible Axion Dark Matter with the Axion Dark Matter Experiment, Phys. Rev. Lett. 120, 151301 (2018).

[94] J. De Miguel-Hernández, A dark matter telescope probing the 6 to $60 \mathrm{GHz}$ band, arXiv:2003.06874.

[95] A. Droster and K. van Bibber (HAYSTAC Collaboration), HAYSTAC status, results, and plans, in 13th Conference on the Intersections of Particle and Nuclear Physics, 2019 (2019), https://inspirehep.net/conferences/ 1655149.

[96] T. Piffl, C. Scannapieco, J. Binney, M. Steinmetz, R.-D. Scholz, M.E. K. Williams, R. S. de Jong, G. Kordopatis, G. Matijevič, O. Bienaymé et al., The rave survey: The galactic escape speed and the mass of the Milky Way, Astron. Astrophys. 562, A91 (2014).

[97] G. Monari, B. Famaey, I. Carrillo, T. Piffl, M. Steinmetz, R. F. G. Wyse, F. Anders, C. Chiappini, and K. Janßen, The escape speed curve of the galaxy obtained from gaia $\mathrm{dr} 2$ implies a heavy Milky Way, Astron. Astrophys. 616, L9 (2018).

[98] A. J. Deason, A. Fattahi, V. Belokurov, N. W. Evans, R. J. J. Grand, F. Marinacci, and R. Pakmor, The local high-velocity tail and the galactic escape speed, Mon. Not. R. Astron. Soc. 485, 3514 (2019).

[99] F.-S. Ling, P. Sikivie, and S. Wick, Diurnal and annual modulation of cold dark matter signals, Phys. Rev. D 70, 123503 (2004).

[100] J. Vergados and Y. Semertzidis, Axionic dark matter signatures in various halo models, Nucl. Phys. B915, 10 (2017). 
[101] C. A. J. O'Hare and A. M. Green, Axion astronomy with microwave cavity experiments, Phys. Rev. D 95, 063017 (2017).

[102] J. W. Foster, N. L. Rodd, and B. R. Safdi, Revealing the dark matter halo with axion direct detection, Phys. Rev. D 97, 123006 (2018).

[103] C. A. O'Hare, C. McCabe, N. W. Evans, G. Myeong, and V. Belokurov, Dark matter hurricane: Measuring the S1 stream with dark matter detectors, Phys. Rev. D 98, 103006 (2018).

[104] N. Bozorgnia, G. B. Gelmini, and P. Gondolo, Daily modulation due to channeling in direct dark matter crystalline detectors, Phys. Rev. D 84, 023516 (2011).

[105] F. Mayet et al., A review of the discovery reach of directional dark matter detection, Phys. Rep. 627, 1 (2016).

[106] A. Caputo, A. J. Millar, and E. Vitagliano, Revisiting longitudinal plasmon-axion conversion in external magnetic fields, Phys. Rev. D 101, 123004 (2020).
[107] H. A. Weldon, Simple rules for discontinuities in finite temperature field theory, Phys. Rev. D 28, 2007 (1983).

[108] J. Kapusta and C. Gale, Finite-Temperature Field Theory: Principles and Applications, Cambridge Monographs on Mathematical Physics (Cambridge University Press, Cambridge, England, 2011), ISBN 9780511535130, https://doi.org/10.1017/CBO9780511535130.

[109] T.-K. Kuo and J. T. Pantaleone, Neutrino oscillations in matter, Rev. Mod. Phys. 61, 937 (1989).

[110] C. Giunti and C. W. Kim, Fundamentals of Neutrino Physics and Astrophysics (Oxford University Press, Oxford, 2007), https://dx.doi.org/10.1093/acprof:oso/ 9780198508717.001.0001.

[111] E. Hardy and R. Lasenby, Stellar cooling bounds on new light particles: Plasma mixing effects, J. High Energy Phys. 02 (2017) 033. 